\title{
A Novel Approach to Post-Traumatic Foot and Ankle Pains using Percutaneous Ultrasound Guided Cryoneurolysis: A Case Report
}

\author{
David H Sherwood, DO, Jason-Flor V Sisante, PhD and Neil A Segal, MD, MS* \\ Department of Rehabilitation Medicine, University of Kansas Medical Center, USA

\begin{abstract}
*Corresponding author: Neil A Segal, MD, MS, Department of Rehabilitation Medicine, University of Kansas Medical Center, 3901 Rainbow Boulevard, Mailstop 1046, Kansas City, KS 66160, USA, Tel: (913)-
\end{abstract} \\ 945-8985
}

\section{Introduction}

Cryoneurolysis is a minimally invasive, low side effect profile, evidence-supported intervention currently with FDA approval to produce lesions in peripheral nervous tissue, including for relief of pain associated with knee osteoarthritis [1]. The mechanism of action on a peripheral nerve is temporary axonal signal disruption via Wallerian degeneration. While the axon and myelin sheath degenerate, the endoneurium, perineurium and epineurium are unaffected. Schwann cells and macrophages clear debris, and secrete growth factors that allow for axonal regrowth. Current osteoarthritis treatment supports efficacy when used along the infrapatellar branches of the saphenous nerve for medial knee pain and anterior femoral cutaneous nerve for superior knee pain [1]. However, cryoneurolysis is limited only by operator knowledge of peripheral nervous anatomy, as other peripheral nerves beyond the knee have been blocked in practice with success $[1,2]$. Cryoneurolysis is both a safe and effective method of pain relief for which novel approaches can be developed.

Valderrabano, et al. estimate that $1 \%$ of the world's population suffers from ankle osteoarthritis [3]. Ankle osteoarthritis most commonly has a post-traumatic etiology. Weatherall, et al. suggested that elderly patients may experience arthritis of the foot or ankle at a rate as high as $50 \%$ [4]. Given this sizable population, there is a need for effective therapies to treat pain and restore function. Data from the Center for Disease Control and
Prevention suggest that foot and ankle arthrodesis as a surgical intervention has been on the rise from 1994 (8.2/100,000 per capita) to 2006 (20.2/100,000 per capita) [5]. Ankle arthrodesis reduces pain but does not completely eliminate it [1]. Furthermore, in a sample of 23 patients who had an isolated ankle arthrodesis for painful post-traumatic arthritis of the ankle and were followed for 12 to 44 years following surgery, the majority had "substantial, and accelerated, arthritic changes" in the operative foot [6]. In these patients, WOMAC, Foot Function Index, and Short Form-36 scores have demonstrated that foot pain limits physical function [6].

While arthrodesis remains the standard of care for end-stage foot and ankle osteoarthritis, there are several drawbacks, namely: chronic post-operative pain, adjacent joint arthritis, and functional limitations [5]. Interventions for these post-operative chronic conditions have been restricted to non-steroidal anti-inflammatory medications, durable medical equipment, orthoses, and injections. All have varying levels of efficacy and side effects. While cryoneurolysis has been used for knee pain, this intervention has yet to be well-described for treatment of foot and ankle osteoarthritis. We identified few reports of cryoneurolysis being offered for foot or ankle pain. Gabriel, et al. outlined a case series of novel uses for cryoneurolysis: A patient with burns to the dorsal and plantar aspect of the right first three toes was treated with cryoneurolysis of the posterior tibial and superficial fibular nerves [7]. It can be deduced from the scant literature that cryoneurol- 
ysis, while an effective treatment for other peripheral nerves, may not be widely considered for treatment of foot and ankle pain. For instance, a four-year retrospective study showed that of 59 cryoneurolysis treatments, 10 were utilized to alleviate sural nerve pain. However, the investigators did not report the efficacy of the sural injections nor the length of symptom relief from sural injections [8]. To advance understanding regarding this potential, we present a case in which pain relief was achieved immediately following sural and superficial fibular cryoneurolysis.

\section{Case Report}

A 73-year-old woman with a past medical history significant for fibromyalgia, coronary artery disease, deep vein thrombosis, and hypertension presented for evaluation of left foot and ankle pain. Onset was 13 years prior, when a cement mixer fell onto her left foot. She reported pain in her "entire ankle", but localized the pain to the dorsal left foot. She characterized the pain as "burning", with radiation up her leg. Weight bearing exacerbated her pain, limiting her quality of life and physical function. She used a single point cane for offloading of her affected foot. Trials of mediations included, but were not limited to: Diclofenac gel, gabapentin, pregabalin, fentanyl patches, and nortriptyline without relief of left foot pain. She could tolerate the pain in her left foot with oxycodone $10 \mathrm{mg} 8 \times$ per day. She completed physical therapy, but without relief. She had previously had placement of a spinal cord stimulator (2015) without relief. She reported 9 reconstructive surgeries of her affected foot and ankle. The surgeries within our hospital system included: left ankle fusion (2007), repeat left ankle fusion (2011), arthrodesis of left great toe metatarsophalangeal joint (2016), sesamoidectomy of the first toe on the left (2016). Upon inspection and palpation, she exhibited pitting edema throughout the left foot and ankle. She could move only the $2^{\text {nd }}, 3^{\text {rd }}, 4^{\text {th }}$, and $5^{\text {th }}$ digits of her left foot. She had zero degrees of dorsiflexion or plantar flexion of the left ankle. She was without neurologic deficits and upper motor neuron signs. She exhibited tenderness and allodynia over the entire left foot with increased tenderness over the lateral malleolus.

Our patient appeared to be suffering from post-traumatic left foot and ankle osteoarthritis likely exacerbated by failed ankle fusion syndrome. Given the patient's lack of successful response to conventional management, we considered intervening with ultrasound-guided cryoneurolysis.

\section{Procedural Methods and Materials}

The superficial fibular and sural nerves were targeted based on the distribution of her pain. She reported pre-procedural pain severity of $8 / 10$. The procedure was carried out under sterile technique, utilizing a sterile ultrasound transducer cover and sterile ultrasound gel. Pre-procedural scanning was performed to determine optimal needle approach for the procedure. The patient was prepared and draped in sterile fashion. The skin superficial to each nerve was prepared with sterile chlorhexidine. The skin at the site was then anesthetized subcutaneously with 5 cc of $1 \%$ lidocaine, using a 1-inch $25 \mathrm{G}$ needle, raising a skin wheal superficial to each nerve. After confirming local anesthesia, a cryoprobe was inserted with ultrasonographic guidance towards the sural nerve lateral to the Achilles tendon and the small saphenous vein. The sterile tip was iteratively inserted, with overlap until the entire treatment area for the sural nerve was completed. The same process was then repeated for the superficial fibular nerve, which was treated approximately $10 \mathrm{~cm}$ proximal to the lateral malleolus in the triangle formed by the peroneus brevis and extensor digitorum longus muscles and the overlying fascia.

\section{Results}

The patient was elated to report post-procedural pain severity of $0 / 10$. She was instructed to follow up in five months, or sooner, if needed. Unfortunately, patient was lost to follow up. However, in a phone call which took place 11 months following the procedure, patient reported she experienced pain relief for "at least several weeks" post-intervention, but was unable to specify the exact chronicity of her relief.

\section{Limitations}

Our results are limited by a lack of distinction on the initial examination between passive and active range of motion per documentation. This information would have provided helpful data points to evaluate in order to further the understanding of the functional benefits of percutaneous ultrasound guided cryoneurolysis. Moreover, as the patient was lost to follow up, we are unable to comment on the specific chronicity of the treatment in her case beyond her immediate post-procedural pain relief.

Two years following the procedure, an EMG demonstrated evidence of a distal left peroneal and tibial neuropathy. Given her history of multiple left foot and ankle surgeries, it is reasonable to assume her neuropathies were post-op complications. While a polyneuropathy could be possible, contralateral sural response was normal. Moreover, there was no radiculopathy, plexopathy, or other abnormal process noted. While this study was not available before the procedure was done, the study may suggest that there was an underlying neuropathy contributing to her pain in conjunction with the post-traumatic left foot and ankle osteoarthritis.

\section{Discussion}

This case demonstrated reduced ankle joint pain using cryoneurolysis of the sural and superficial fibular nerves under ultrasound guidance. This treatment 


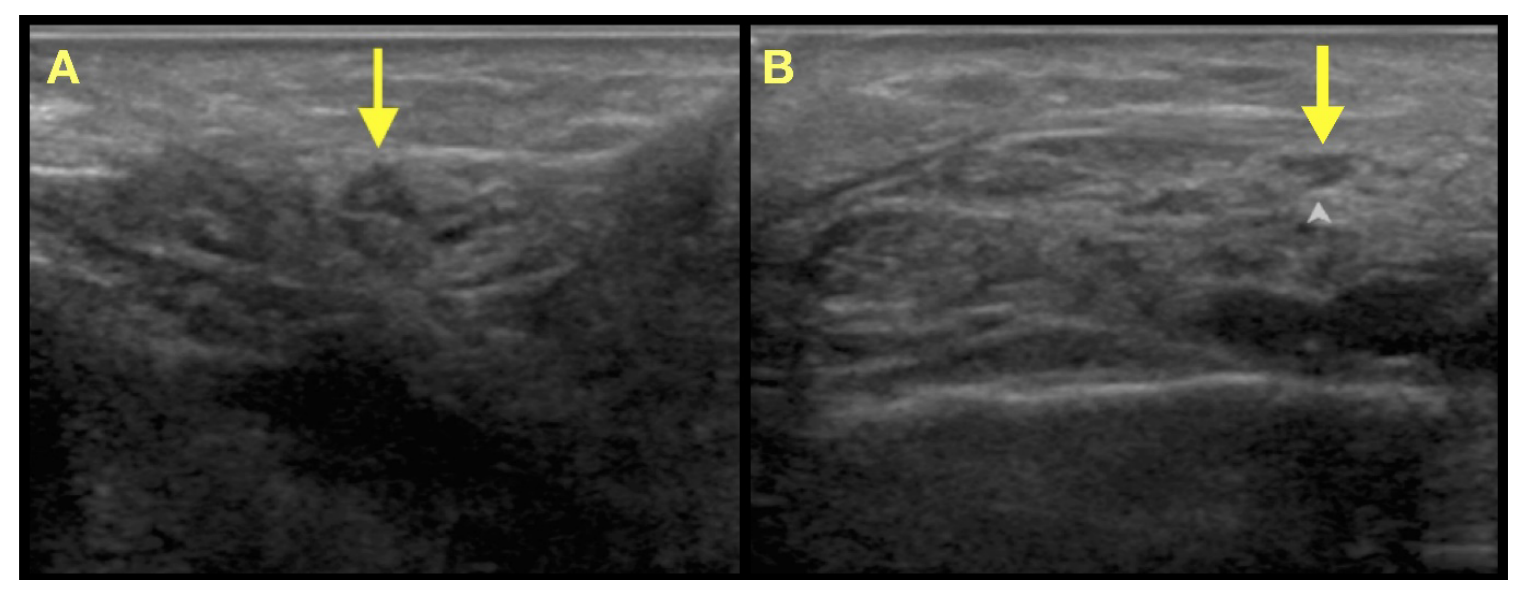

Figure 1: Ultrasonographic images of nerves localized: A) left superficial fibular nerve deep to fascia; B) left sural nerve lateral to Achilles' tendon.

has been reported to create a reversible nerve block through inducing an axonotmetic nerve injury. When sensory nerves are treated, afferent conduction temporarily ceases, immediately alleviating pain. Because the endoneurium, perineurium and epineurium remain intact, myelinated axons regenerate at a rate of 1.0$1.5 \mathrm{~mm}$ per day. Thus, the duration of the analgesia depends on the distance from the distal end of the terminal axon.

Based on current evidence related to the infrapatellar branches of the saphenous nerve, patients can expect a treatment effect of 120-150 days [1]. However, this Figure 1 is variable based on the peripheral nerve treated. This case is consistent with other reports in supporting that cryoneurolysis is attractive from both patient and provider standpoints - it is minimally invasive, has a favorable side-effect profile, can be offered on an outpatient basis, and has demonstrated efficacy in contexts in which other interventions have not. This case provides additional evidence that cryoneurolysis has a potential growing role in the treatment of pain due to osteoarthritis and related etiologies $[1,2,7]$.

We demonstrated that the expansion of cryoneurolysis as a modality for treating pain is limited only by the operator's ability to identify the sensory nerve via imaging guidance. Future efforts on this treatment modality should focus on the chronicity of pain relief for the superficial fibular and sural nerves, and on function- al outcomes.

\section{References}

1. Radnovich R, Scott D, Patel AT, Olson R, Dasa V, et al. (2017) Cryoneurolysis to treat the pain and symptoms of knee osteoarthritis: A multicenter, randomized, double-blind, sham-controlled trial. Osteoarthritis Cartilage 25: 1247-1256.

2. Trescot AM (2003) Cryoanalgesia in interventional pain management. Pain Physician 6: 345-360.

3. Valderrabano V, Horisberger M, Russell I, Dougall H, Hintermann B (2009) Etiology of ankle osteoarthritis. Clin Orthop Relat Res 467: 1800-1806.

4. Weatherall JM, Mroczek K, McLaurin T, Ding B, Tejwani N (2013) Post-traumatic ankle arthritis. Bull Hosp Jt Dis 71: 104-112.

5. Best MJ, Buller LT, Miranda A (2015) National trends in foot and ankle arthrodesis: 17-Year analysis of the national survey of ambulatory surgery and national hospital discharge survey. J Foot Ankle Surg 54: 1037-1041.

6. Coester LM, Saltzman CL, Leupold J, Pontarelli W (2001) Long-term results following ankle arthrodesis for post-traumatic arthritis. J Bone Joint Surg Am 83: 219-228.

7. Gabriel RA, Finneran JJ, Asokan D, Trescot AM, Sandhu NS, et al. (2017) Ultrasound-guided percutaneous cryoneurolysis for acute pain management: A Case Report. A A Case Rep 9: 129-132.

8. Walter WR, Burke CJ, Adler RS (2017) Ultrasound-guided therapeutic injections for neural pathology about the foot and ankle: A 4 year retrospective review. Skeletal Radiol 46: 795-803. 